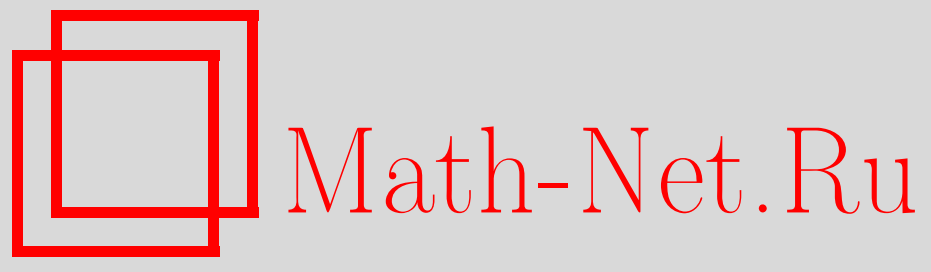

А. В. Словеснов, Предельное интегральное кручение замкнутых аналитических кривых, Матем. заметки, 2011, том 89, выпуск 1, 153-156

DOI: https://doi.org/10.4213/mzm8929

Использование Общероссийского математического портала Math-Net.Ru подразумевает, что вы прочитали и согласны с пользовательским соглашением http://www . mathnet.ru/rus/agreement

Параметры загрузки:

IP : 54.224 .60 .19

26 апреля 2023 г., 04:50:10

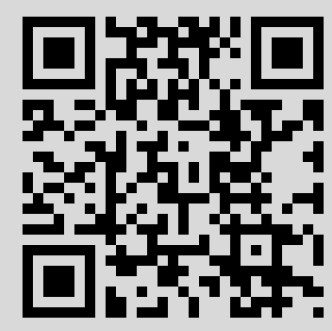




\section{Предельное интегральное кручение замкнутых аналитических кривых}

\section{А. В. Словеснов}

Рассмотрим некоторую пространственную ${ }^{1}$ замкнутую аналитическую кривую с радиус-вектором $r(t), t \in[0, T]$, и плоскость П с единичной нормалью $e$. Определим сжатие кривой $r(t)$ на плоскость П с помощью семейства $\left\{r_{\lambda}(t), \lambda \in[0,1]\right\}$, где

$$
r_{\lambda}(t)=r(t)-(1-\lambda)(e, r(t)) e, \quad t \in[0, T] .
$$

Пусть также $\varkappa_{\text {int }}(\lambda)$ - интегральное кручение кривой $r_{\lambda}(t)$ :

$$
\varkappa_{\text {int }}(\lambda)=\int_{0}^{T} \frac{\left\langle r_{\lambda}^{\prime}(t), r_{\lambda}^{\prime \prime}(t), r_{\lambda}^{\prime \prime \prime}(t)\right\rangle}{\left|\left[r_{\lambda}^{\prime}(t), r_{\lambda}^{\prime \prime}(t)\right]\right|^{2}}\left|r_{\lambda}^{\prime}(t)\right| d t .
$$

Тогда предельным интегралъным кручением кривой $r(t)$ при сжатии на плоскость П назовем величину $\lim _{\lambda \rightarrow 0+0} \varkappa_{\text {int }}(\lambda)$. Ниже мы покажем существование этого предела, вычислив его значение. Для этого введем обозначения для подвижного репера Френе кривой $r(t)-\{\tau(t), \nu(t), \beta(t)\}-$ и заметим, что

$$
\varkappa_{\text {int }}(\lambda)=\lambda \int_{0}^{T} \varkappa(t) \frac{\sqrt{\sin ^{2} \psi(t)+\lambda^{2} \cos ^{2} \psi(t)}}{\cos ^{2} \varphi(t)+\lambda^{2} \sin ^{2} \varphi(t)}\left|r^{\prime}(t)\right| d t,
$$

где $\psi(t)$ - это угол между векторами $\tau(t)$ и $e$, а $\varphi(t)$ - угол между $\beta(t)$ и $e$. Из (1) видно, что если направление $e$ таково, что всюду на кривой $\cos \varphi(t) \neq 0$, то

$$
\lim _{\lambda \rightarrow 0+0} \varkappa_{i n t}(\lambda)=0
$$

а в общем случае при его вычислении необходимо рассмотреть интегралы по сколь угодно малым окрестностям точек $t \in[0, T]$, в которых функция $\cos \varphi(t)$ обращается в ноль. Условимся называть такие точки особыми и отметим, что в силу аналитичности кривой, а также предположения $\varkappa(t) \not \equiv 0$, число особых точек конечно.

Допустим, что хотя бы одна особая точка $t_{0}$ кривой $r(t)$ для плоскости П существует, и определим ее вклад в предельное интегральное кручение. Предположив без потери общности, что $t_{0}=0$, рассмотрим интеграл $(1)$ по окрестности $(-\delta, \delta)$ в пределе при $\lambda \rightarrow 0+0$, считая число $\delta>0$ сколь угодно малым. Наша ближайшая цель состоит в том, чтобы последовательно упростить подынтегральную функцию до такой степени, чтобы интеграл (1) можно было вычислить.

Введем следующие величины:

$$
\begin{aligned}
& \Delta_{1}(\lambda, \delta)=\lambda \int_{-\delta}^{\delta} \varkappa(t) \frac{\sqrt{\sin ^{2} \psi(t)+\lambda^{2} \cos ^{2} \psi(t)}-|\sin \psi(t)|}{\cos ^{2} \varphi(t)+\lambda^{2} \sin ^{2} \varphi(t)}\left|r^{\prime}(t)\right| d t, \\
& \Delta_{2}(\lambda, \delta)=\lambda \int_{-\delta}^{\delta} \varkappa(t)\left(\frac{|\sin \psi(t)|}{\cos ^{2} \varphi(t)+\lambda^{2} \sin ^{2} \varphi(t)}-\frac{|\sin \psi(t)|}{\cos ^{2} \varphi(t)+\lambda^{2}}\right)\left|r^{\prime}(t)\right| d t .
\end{aligned}
$$

Нетрудно показать, что $\left|\Delta_{1}(\lambda, \delta)\right| \leqslant C \cdot \delta$, где $C$ - некоторая абсолютная постоянная, и $\lim _{\lambda \rightarrow 0+0} \Delta_{2}(\lambda, \delta)=0$. Отсюда следует, что вклад особой точки $t=0$ в предельное интегральное кручение составляет

$$
\lim _{\lambda \rightarrow 0+0} \lambda \int_{-\delta}^{\delta} \varkappa(t) \frac{|\sin \psi(t)|}{\cos ^{2} \varphi(t)+\lambda^{2}}\left|r^{\prime}(t)\right| d t .
$$

Работа выполнена при поддержке Минобрнауки РНП (грант № 2.1.1.3704)

${ }^{1}$ Под словом пространственная мы понимаем кривую с кручением $\varkappa \neq \equiv$.

(C) А. В. Словеснов, 2011 
Для вычисления последнего предела сделаем следующее преобразование. Обозначив буквой $s$ натуральный параметр кривой, с помощью формул Френе находим

$$
(\cos \varphi(t))_{t}^{\prime}=(\beta(t), e)_{t}^{\prime}=\left(\beta_{t}^{\prime}(t), e\right)=\left(\beta_{s}^{\prime}(t) \cdot s_{t}^{\prime}, e\right)=\varkappa(t)\left|r^{\prime}(t)\right| \cdot(\nu(t), e) .
$$

Таким образом, интересующий нас предел можно переписать в виде

$$
\lim _{\lambda \rightarrow 0+0} \lambda \int_{-\delta}^{\delta} \frac{|\sin \psi(t)|}{(\nu(t), e)} \cdot \frac{(\cos \varphi(t))_{t}^{\prime}}{\cos ^{2} \varphi(t)+\lambda^{2}} d t
$$

Покажем, что функция $|\sin \psi(t)| /(\nu(t), e)$ в точке $t=0$ имеет односторонние пределы, равные 1 или -1 . Из определения особой точки следует, что вектор е лежит в плоскости $\langle\tau(0), \nu(0)\rangle$. Поскольку векторы $\tau(0)$ и $\nu(0)$ ортонормированы, то $|\sin \psi(0)|=|(\nu(0), e)|$, и в случае $(\nu(0), e) \neq 0$ существование односторонних пределов очевидно. При $(\nu(0), e)=0$ рассмотрим равенство

$$
\begin{aligned}
(\tau(t), e)^{2}+(\nu(t), e)^{2}+(\beta(t), e)^{2} \equiv 1 & \Longleftrightarrow \cos ^{2} \varphi(t)+(\nu(t), e)^{2}=\sin ^{2} \psi(t) \\
& \Longleftrightarrow(\nu(t), e)^{2}\left(1+(\cos \varphi(t) /(\nu(t), e))^{2}\right)=\sin ^{2} \psi(t) .
\end{aligned}
$$

Как видно из (2), второе слагаемое в скобках есть бесконечно малая величина при $t \rightarrow 0$, откуда и следует существование указанных односторонних пределов, равных \pm 1 . С учетом доказанного мы можем ввести следующие обозначения:

$$
s_{1}:=\lim _{t \rightarrow 0-0} \frac{|\sin \psi(t)|}{(\nu(t), e)}, \quad s_{2}:=\lim _{t \rightarrow 0+0} \frac{|\sin \psi(t)|}{(\nu(t), e)} .
$$

Покажем, что предел (3) можно переписать в виде

$$
s_{1} \cdot \lim _{\lambda \rightarrow 0+0} \lambda \int_{-\delta}^{0} \frac{(\cos \varphi(t))_{t}^{\prime}}{\cos ^{2} \varphi(t)+\lambda^{2}} d t+s_{2} \cdot \lim _{\lambda \rightarrow 0+0} \lambda \int_{0}^{\delta} \frac{(\cos \varphi(t))_{t}^{\prime}}{\cos ^{2} \varphi(t)+\lambda^{2}} d t .
$$

Действительно, число $\delta$ мы вправе выбирать произвольным, поэтому справедливость этого разложения в сумму оправдывает оценка

$$
\lambda \int_{-\delta}^{\delta}|t| \cdot \frac{\left|(\cos \varphi(t))^{\prime}\right|}{\cos ^{2} \varphi(t)+\lambda^{2}} d t \leqslant C \cdot \delta .
$$

Последняя оценка, как нетрудно проверить, следует из представления аналитической функции $\cos \varphi(t)$ в окрестности точки $t=0: \cos \varphi(t) \sim A \cdot t^{k}, k \geqslant 1$. Пределы из формулы (5) легко вычисляются:

$$
\begin{aligned}
& \lim _{\lambda \rightarrow 0+0} \lambda \int_{-\delta}^{0} \frac{(\cos \varphi(t))_{t}^{\prime}}{\cos ^{2} \varphi(t)+\lambda^{2}} d t=\frac{\pi}{2} \operatorname{sgn}\left((\cos \varphi(t))_{t}^{\prime}\right)_{t \in(-\delta, 0)}, \\
& \lim _{\lambda \rightarrow 0+0} \lambda \int_{0}^{\delta} \frac{(\cos \varphi(t))^{\prime}}{\cos ^{2} \varphi(t)+\lambda^{2}} d t=\frac{\pi}{2} \operatorname{sgn}\left((\cos \varphi(t))_{t}^{\prime}\right)_{t \in(0, \delta)} .
\end{aligned}
$$

Учитывая равенства (2) и (4), заключаем, что вклад особой точки $t=0$ в предельное интегральное кручение равен

$$
\frac{\pi}{2}\left(\operatorname{sgn}(\varkappa(t))_{t \in(-\delta, 0)}+\operatorname{sgn}(\varkappa(t))_{t \in(0, \delta)}\right) .
$$

Таким образом, доказана

Лемма 1. Вклад особой точки $t=t_{0}$ в предельное интегральное кручение равен 0 , если кручение в этой точке равно 0 и его знак меняется при переходе через эту точку. $B$ противном случае ее вклад составляет $\pi \cdot \operatorname{sgn} \varkappa(t)$, где точка $t \neq t_{0}$ достаточно близка $\kappa t_{0}$. 
Поскольку мы работаем с аналитическими кривыми и особых точек, как было отмечено выше, лишь конечное число, справедлива

ТЕорема 1. Предельное интегральное кручение замкнутой аналитической кривой при сжатии на любую плоскость кратно числу $\pi$.

ЗАмечАниЕ 1. Во-первых, отметим, что при проектировании на разные плоскости могут получаться разные значения предельного интегрального кручения, сохраняется лишь их кратность числу $\pi$. Во-вторых, нетрудно показать, что любое число, кратное $\pi$, может выступать в качестве предельного интегрального кручения некоторой кривой при сжатии на некоторую плоскость. Для этого достаточно рассмотреть кривую 2 с радиус-вектором

$$
\rho_{m}(t)=\left(\sin t,(1-\cos t)^{3},(1-\cos t) \sin t\right), \quad t \in[0,2 \pi m], \quad m \in \mathbb{N} .
$$

Нетрудно проверить, что ее предельное интегральное кручение при сжатии на плоскость Оху равно $-\pi m$.

Теорема 1 справедлива для всех кривых и всех плоскостей сжатия. Ниже мы уточним полученное утверждение, разбив кривые на два класса и исключив некоторые плоскости сжатия. Согласно работе Сабитова [2] все замкнутые аналитические кривые можно разбить на два непересекающихся класса ориентируемых и полуориентируемых кривых в зависимости, соответственно, от периодичности или антипериодичности аналитических векторов главной нормали и бинормали. Чтобы выявить различие между поведением интегрального кручения у кривых разных классов, мы наложим некоторое ограничение на плоскость сжатия, оставаясь в случае общего положения. Мы будем считать, что выбранный вектор $e$, ортогональный плоскости сжатия, не коллинеарен ни одному из векторов $\{\tau(t), t \in[0, T]\}$. В этом случае мы будем называть плоскость сжатия регулярной. Если вектор $e$ понимать как точку на единичной сфере, то данное требование исключает из рассмотрения те направления $e$, которые принадлежат кривой $\tau(t), t \in[0, T]$, расположенной на этой сфере, т. е. множество направлений двумерной меры 0. Таким образом, указанное ограничение соответствует случаю общего положения.

ТЕорема 2. Предельное интегралъное кручение замкнутой аналитической кривой при сжатии на регулярную плоскость кратно числу $\pi$ с четным коэфбициентом пропорииональности, если кривая периодическая, и пропориионально $\pi$ с нечетным коэфбичиентом пропорчиональности, если кривая полупериодическая.

ДокАЗАтЕЛЬство. Зафиксируем регулярную плоскость сжатия П и рассмотрим некоторую особую точку $t_{0}$. Сразу отметим, что отсутствие таких точек означает, что мы работаем с ориентируемой кривой, а предельное интегральное кручение равно 0, что согласуется с формулировкой теоремы. Согласно лемме 1 вклад особой точки $t_{0}$ в предельное интегральное кручение определяется поведением кручения в окрестности этой точки. Поскольку мы рассматриваем регулярную плоскость, то в точке $t_{0}$ выполняется неравенство $\left(\nu\left(t_{0}\right), e\right) \neq 0$. Поэтому из формулы $(2)$ следует, что вклад одной особой точки $t_{0}$ составляет $\pm \pi$, если график функции $\cos \varphi(t)=(\beta(t), e)$ пересекает ось абсцисс, и равен 0, если он касается оси абсцисс. В случае ориентируемой кривой указанная функция является периодической и пересекает ось абсцисс четное число раз. Для полуориентируемой кривой эта функция, будучи антипериодической, пересекает ось абсцисс нечетное число раз, откуда и следует утверждение теоремы.

ЗАмечАниЕ 2. Зафиксируем $m \in \mathbb{N}$ и рассмотрим кривую (6), которая при четных $m$ является ориентируемой, а при нечетных - полуориентируемой. Как отмечалось в замечании к теореме 1 , ее предельное интегральное кручение при сжатии на плоскость $O x y$

\footnotetext{
2Эта кривая была построена Г. Шварцем в работе [1] при изучении аналитических лент Мебиуса.
} 
равно $-\pi m$. Если вычислить предельное кручение той же кривой при сжатии на плоскость $O x z$, то оно окажется равным $\pi m$. Это значит, что меняя параметр и направление сжатия, можно получить кривую с любым интегральным кручением из интервала $(-\pi m, \pi m)$. Так как $m$ - произвольное натуральное число, последнее, в свою очередь, позволяет говорить о существовании кривой (как ориентируемой, так и полуориентируемой) с любым наперед заданным интегральным кручением.

Автор выражает благодарность И. Х. Сабитову за постановку задачи и многочисленные консультации.

\section{СПИСОК ЦИТИРОВАННОЙ ЛИТЕРАТУРЫ}

[1] G. Schwarz, Pacific J. Math., 143:1 (1990), 195-200. [2] И. Х. Сабитов, Изв. РАН. Сер. матем., 71:5 (2007), 197-224.

\section{А. В. Словеснов}

Поступило

Московский государственный

университет им. М. В. Ломоносова

E-mail: alexslovesnov@narod.ru 